\title{
OPTIMAL DISTRIBUTED CONTROL OF STOCHASTIC ELLIPTIC SYSTEMS WITH CONSTRAINTS
}

\author{
A. S. Okb El Bab ${ }^{1}$, Abd-Allah Hyder ${ }^{2}$ and A. M. Abdallah ${ }^{3}$ \\ ${ }^{1}$ Department of Mathematics, Faculty of Science, \\ Al-Azhar University, Nasr City, Cairo, Egypt. \\ ahmedokbelbab@yahoo.com \\ ${ }^{2}$ Department of Engineering Mathematics and Physics, Faculty of Engineering, \\ Al-Azhar University, Cairo, Egypt. \\ abdallah.hyder@yahoo.com \\ ${ }^{3}$ Department of Basic Science, Higher Technological Institute, \\ Tenth of Ramadan, Egypt. \\ ahmedabdallah6236@yahoo.com
}

\begin{abstract} Neumann problems.

\section{SUBJECT CLASSIFICATION: 35J47, 60H40, 93E20. \\ KEYWORDS: Elliptic Systems; White Noise; Stochastic Control. INTRODUCTION}

The objective of this paper is to study the optimality for stochastic non cooperative elliptic systems. A distributed control problem for a stochastic elliptic systems with constraints on states and controls is studied. First, the existence and uniqueness of the state process for these systems are proved. The necessary and sufficient conditions of optimality are derived for the Dirichlet and

Stochastic systems play an important role in mathematical models of phenomena in many fields of science, engineering, finance, biology, epidemiology and economics. In view probability theory, state of a stochastic system is randomly determined. Recently, Many researchers have been directed to the studies of optimal control of stochastic systems due to their last importance. They deal with stochastic differential equations (SDEs) $[6,16]$.

Zhou was one of the first scientists to have used stochastic partial differential equations (SPDEs). He developed the necessary conditions of optimality (maximum principle) for a very strictly elliptic second order partial differential equations on a $d$ -dimensional space [15]. The theory of backward and forward partial differential equations (PDEs) has been developed quickly, and it became a powerful tool in the study of PDEs. Moreover, backward and forward SPDEs appeared and was studied in many literatures (see e.g. [1,3]). The optimality for SPDEs is discussed in [15]. There are difficulties in the discussion of the solution methods of systems of forward and backward SPDEs. So, the unique method for the solution is the maximum principle. Researchers used the same steps; they discussed the maximum principle to face the difficulties of investigating the solutions.

Our first interest in this work is to implement new strategies that give a full study of the stochastic control problems. Firstly, we derive the optimality conditions. Secondly, we apply the easiest methods to simplify the technique of the stochastic control problem. Finally, we develop the study of the stochastic optimal control without constraints on the stochastic systems and without forward and backward SPDEs method. In this paper, we discuss the optimal control problem for $2 \times 2$ stochastic non cooperative elliptic systems with Dirichlet and Neumann conditions. We give the unique solution for these systems (the state process of the system). Then after, we formulate the control problem and proved the existence and uniqueness of the control. Also, we present the set of equations and inequalities that characterizes the distributed control for these systems. Moreover, the optimal control problem of the generalized $N \times N$ stochastic Neumann elliptic systems with constraints is studied. In all our considered problems the control of distributed type with state constraints is discussed.

This paper is organized as follows: In section 1; we introduce the basic notations and some function spaces defined on $R^{1}$. Section 2 is devoted to study the optimal control for $2 \times 2$ stochastic elliptic systems with Dirichlet conditions. In section 3 , we also study the problem with Neumann conditions and we introduce the main results of this paper.

\section{Notations}

This section covers the basic definitions and notations, which are necessary to present our work. Let $G$ be a nonempty open set in $\mathrm{R}^{n}$, the function $\phi: G \rightarrow \mathrm{R}$ is said to have a compact support if there exists a compact subset $K$ of $G$ such that $\phi(x)=0$ 
$\forall x \in G \backslash K$. Let $D(G)$ be the space of infinitely differentiable functions $\phi$ with compact support (space of test functions). The space of distributions is denoted by $D^{\prime}(G), D(G)$ is dense in $L^{2}(G)$ and $D(G) \subset L^{p}(G) \subset D^{\prime}(G)$.

For a multi-index $\alpha=\left(\alpha_{1}, \alpha_{2}, \ldots, \alpha_{n}\right) \in \mathrm{N}$, where $\alpha_{i}$ are non negative integers and $\Phi \in D(G)$, we define

$$
D^{\alpha}(\Phi(x))=\frac{\partial^{|\alpha|} \Phi}{\partial x_{1}^{\alpha_{1}} \partial x_{2}^{\alpha_{2}} \ldots \partial x_{n}^{\alpha_{n}}}(x), x=\left(x_{1}, x_{2}, \ldots, x_{n}\right)
$$

where $|\alpha|=\sum_{i=1}^{n} \alpha_{i}$. The partial derivative $\partial^{|\alpha|} T$ of a distribution $T$ is defined by

$$
<\partial^{\alpha} T, \Phi>=(-1)^{|\alpha|}<\partial^{\alpha} \Phi, T>, \Phi \in D(G), T \in D^{\prime}(G) .
$$

Let $(\Omega, \mathrm{F}, P)$ be a probability space, where $\Omega$ is a sample space, $\mathrm{F}$ is an $\sigma$-algebra and $P$ is a probability measure. We introduce

$$
L^{2}(\Omega, \mathrm{F}, P ; G)=\left\{u: G \times \Omega \rightarrow \mathrm{R} \mid u \text { ismeasurableand } \int_{\Omega} \mathrm{P} u \mathrm{P}^{2} d p<\infty\right\},
$$

with inner product $(., .)_{L^{2}(\Omega, \mathrm{F}, P ; G)}: L^{2}(\Omega, \mathrm{F}, P ; G) \times L^{2}(\Omega, \mathrm{F}, P ; G) \rightarrow \mathrm{R}$, defined as

$$
(u, v)_{L^{2}(\Omega, F, P ; G)}=\left(\int_{\Omega}\left(\int_{G} \nabla u(x) \nabla v(x) d x\right) d p\right)
$$

The Sobolev space of order $m$ [4], denoted by $H^{m}(\Omega, F, P ; G)$, is defined by

$$
H^{m}(\Omega, \mathrm{F}, P ; G)=\left\{v \in L^{2}(\Omega, \mathrm{F}, P ; G), \frac{\partial^{m} v}{\partial x_{i}^{m}} \in L^{2}(\Omega, \mathrm{F}, P ; G), 1 \leq i \leq n\right\},
$$

with respect to the inner product

$$
<f, g>_{H^{m}(\Omega, F, P ; G)}=\sum_{|\alpha| \leq m}<D^{\alpha} f, D^{\alpha} g>_{L^{2}(\Omega, F, P ; G)},
$$

where $\frac{\partial v}{\partial x_{i}}$ are the derivative in the sense of distribution.

An importance subspace of $H^{m}(\Omega, \mathrm{F}, P ; G)$ is given by

$$
H_{0}^{m}(\Omega, \mathrm{F}, P ; G)=\left\{v \in L^{2}(\Omega, \mathrm{F}, P ; G), \frac{\partial^{\alpha} v}{\partial x_{i}^{\alpha}}=0,|\alpha| \leq m-1\right\} .
$$

The dual space of $H_{0}^{m}(\Omega, \mathrm{F}, P ; G)$ is denoted by $H^{-m}(\Omega, \mathrm{F}, P ; G)$, thus we have the chain;

$$
H_{0}^{m}(\Omega, \mathrm{F}, P ; G) \subseteq L^{2}(\Omega, \mathrm{F}, P ; G) \subseteq H^{-m}(\Omega, \mathrm{F}, P ; G) .
$$

Let $H$ be a Hilbert space. The bilinear form $a: H \times H \rightarrow \mathrm{R}$ is said to be coercive if and only if there exists a constant $c>0$

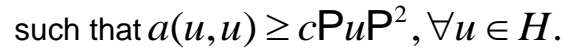

Let $V$ be a Hilbert space on $\mathrm{R}$ and $V^{\prime}$ be its dual space. The Lax- Milgram lemma [12] states: if the bilinear form $a(u, v)$ satisfies the continuity and coerciveness conditions for $u, v \in V$ and the linear form $f \in V^{\prime}$ satisfies the continuity condition for the system $A u=f$ with model $A$, then there exists a unique solution $u$ for the system $A u=f$ such that $a(u, v)=f(v)$. 


\section{2. $2 \times 2$ Stochastic Elliptic Systems with Constraints and Dirichlet Conditions}

In this section, we study the optimal control problem for non cooperative $2 \times 2$ stochastic elliptic systems with Dirichlet Condition:

$$
\left\{\begin{array}{cc}
-\Delta u_{1}(x)=W_{1}(x)-a u_{1}(x)+b u_{2}(x) & x \in G_{1} \\
-\Delta u_{2}(x)=W_{2}(x)-b u_{1}(x)-a u_{2}(x) & x \in G_{2} \\
u_{1}(x)=0, u_{2}(x)=0 & \text { on } \partial G,
\end{array}\right.
$$

with constraints

$$
\left\{\begin{array}{cc}
\left\{\frac{\partial u_{k}(x)}{\partial V_{A}}\right\}^{ \pm}=t\left[u_{k}(x)\right] & \text { on } \gamma \\
=0 & \text { on } \gamma \\
{\left[\frac{\partial u_{k}(x)}{\partial V_{A}}\right]=0} & \text { on } \gamma \\
\frac{\partial u_{k}(x)}{\partial V_{A}}=\sum_{i, j=1}^{n} \frac{\partial u_{k}(x)}{\partial x_{j}} \cos \left(v, x_{i}\right) & k=1,2
\end{array}\right.
$$

where $a, b \in \mathrm{R}^{+}$and $G_{1}$ and $G_{2}$ are two bounded, continous and strictly domains in $\mathrm{R}^{n}$ such that $G_{1} \bigcap G_{2}=\varphi$. Here, $G=G_{1} \bigcup G_{2}$ with boundary $\partial G=\left(\partial G_{1} \bigcup \partial G_{2}\right) \backslash \gamma, \gamma=\partial G_{1} \bigcap \partial G_{2} \neq \varphi, \cos \left(v, x_{i}\right)=i$ th direction cosine of $v, v$ being the normal at $\gamma,[u]=u^{+}-u^{-}$, where $u^{+}=\{u\}^{+}=u(x)$ under $x \in \partial G_{2} \bigcap \gamma, u^{-}=\{u\}^{-}=u(x)$ under $x \in \partial G_{1} \bigcap \nu$, and

$$
0 \leq t \leq t(x) \leq v, t \in C(\gamma), v \text { isaconstant.(2.3) }
$$

While $u(x)=\left(u_{1}(x), u_{2}(x)\right) \in H_{0}^{1}(\Omega, \mathrm{F}, P ; G)$ is a state process and $W(x)=\left(W_{1}(x), W_{2}(x)\right)$ is a brownian motion (wiener process). In the following subsection, we present the existence and uniqueness of (2.1).

\subsection{Existence and Uniqueness for Solution}

In this subsection, we study the existence and uniqueness of solution for (2.1), (2.2). Since

$$
H_{0}^{1}(\Omega, \mathrm{F}, P ; G) \subseteq L^{2}(\Omega, \mathrm{F}, P ; G) \subseteq H^{-1}(\Omega, \mathrm{F}, P ; G),
$$

then we have chain

$$
\left[H_{0}^{1}(\Omega, \mathrm{F}, P ; G)\right]^{2} \subseteq\left[L^{2}(\Omega, \mathrm{F}, P ; G)\right]^{2} \subseteq\left[H^{-1}(\Omega, \mathrm{F}, P ; G)\right]^{2} .
$$

We define the following bilinear form on the sobolev space $\left[H_{0}^{1}(\Omega, \mathrm{F}, P ; G)\right]^{2}$, 


$$
\begin{gathered}
b(u, \Phi)=\mathrm{E}\left(\int_{G}\left(-\Delta u_{1}(x) \Phi_{1}(x)+a u_{1}(x) \Phi_{1}(x)-b u_{2}(x) \Phi_{1}(x)\right) d x\right) \\
+\mathrm{E}\left(\int_{G}\left(-\Delta u_{2}(x) \Phi_{2}(x)+b u_{1}(x) \Phi_{2}(x)+a u_{2}(x) \Phi_{2}(x)\right) d x\right) \\
+\mathrm{E}\left(\int_{\gamma} t\left[u_{1}(x)\right]\left[\Phi_{1}(x)\right]+t\left[u_{2}(x)\right]\left[\Phi_{2}(x)\right] d \gamma\right),(2.4)
\end{gathered}
$$

the linear form is given by

$$
L(\Phi)=\mathrm{E}\left(\int_{G} W_{1}(x) \Phi_{1}(x)+W_{2}(x) \Phi_{2}(x) d x\right)(2.5)
$$

where $W_{1}(x)$ and $W_{2}(x)$ are the components of the wiener process. By Lax-Milgram lemma, we prove the following theorem.

Theorem 2.1. The bilinear form (2.4) satisfies the stochastic coerciveness condition and then there exists a unique solution $u \in\left[H_{0}^{1}(\Omega, \mathrm{F}, P ; G)\right]^{2}$ of system (2.1), conversely, if there exists a unique solution $u \in\left[H_{0}^{1}(\Omega, \mathrm{F}, P ; G)\right]^{2}$ such that $b(u, \Phi)=L(\Phi)$, then we get the system (2.1)

Proof. The bilinear form (2.4) can be written as

$$
\begin{aligned}
& b(u, \Phi)=\mathrm{E}\left(\int_{G}\left(-\Delta u_{1}(x) \Phi_{1}(x)+a u_{1}(x) \Phi_{1}(x)-b u_{2}(x) \Phi_{1}(x)\right) d x\right) \\
& +\mathrm{E}\left(\int_{G}\left(-\Delta u_{2}(x) \Phi_{2}(x)+a u_{2}(x) \Phi_{2}(x)+b u_{1}(x) \Phi_{2}(x)\right) d x\right) \\
& +\mathrm{E}\left(\int_{\gamma}\left(t\left[u_{1}(x)\right]\left[\Phi_{1}(x)\right]+t\left[u_{2}(x)\right]\left[\Phi_{2}(x)\right]\right) d \gamma\right)
\end{aligned}
$$

Applying Green's formula, gives

$$
\begin{aligned}
& b(u, u)=\mathrm{E}\left(\int_{G}\left(\left|\nabla u_{1}(x)\right|^{2}+a\left(u_{1}(x)\right)^{2}-b u_{2}(x) u_{1}(x)\right) d x\right) \\
& +\mathrm{E}\left(\left|\nabla u_{2}(x)\right|^{2}+b u_{1}(x) u_{2}(x)+a\left(u_{2}(x)\right)^{2}\right) \\
& +\mathrm{E}\left(\int_{\gamma}\left(t\left[u_{1}(x)\right]^{2}+t\left[u_{2}(x)\right]^{2}\right) d \gamma\right) \\
& \geq \mathrm{P}_{1} \mathrm{P}_{\left(H_{0}^{1}(\Omega, F, P ; G)\right)^{2}}^{2}+\mathrm{P}_{2} \mathrm{P}_{\left(H_{0}^{1}(\Omega, F, P ; G)\right)^{2}}^{2}
\end{aligned}
$$

Therefore,

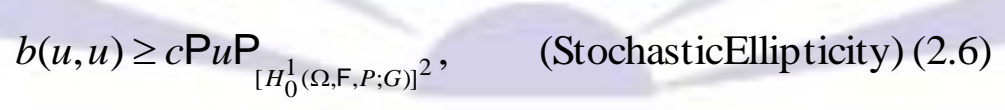

where $c=$ const and $u=\left(u_{1}, u_{2}\right)$. Since the bilinear form satisfies the continuity condition and the linear form is also continuous on $\left[H_{0}^{1}(\Omega, \mathrm{F}, P ; G)\right]^{2}$, then by Lax-Milgram lemma, there exists a unique solution $u=\left(u_{1}, u_{2}\right) \in\left[H_{0}^{1}(\Omega, \mathrm{F}, P ; G)\right]^{2}$, suchthat $b(u, \Phi)=L(\Phi), \forall \Phi \in\left[H_{0}^{1}(\Omega, \mathrm{F}, P ; G)\right]^{2}$. Conversely, $\quad$ when $b(u, \Phi)=L(\Phi), \forall \Phi \in\left[H_{0}^{1}(\Omega, \mathrm{F}, P ; G)\right]^{2}$ and $u=\left(u_{1}, u_{2}\right) \in\left[H_{0}^{1}(\Omega, \mathrm{F}, P ; G)\right]^{2}$, we have

$$
\begin{aligned}
& \mathrm{E}\left(\int_{G}\left(\nabla u_{1}(x) \nabla \Phi_{1}(x)+a u_{1}(x) \Phi_{1}(x)-b u_{2}(x) \Phi_{1}(x)\right) d x\right) \\
& +\mathrm{E}\left(\int_{G}\left(\nabla u_{2}(x) \nabla \Phi_{2}(x)+b u_{1} \Phi_{2}(x)+a u_{2}(x) \Phi_{2}(x)\right) d x\right)
\end{aligned}
$$




$$
\begin{aligned}
& +\mathrm{E}\left(\int_{\gamma}\left(t\left[u_{1}(x)\right]\left[\Phi_{1}(x)\right]+t\left[u_{2}(x)\right]\left[\Phi_{2}(x)\right]\right) d \gamma\right) \\
& +\mathrm{E}\left(\int_{\partial G}\left(F\left(u_{1}(x), \Phi_{1}(x)\right)+H\left(u_{2}(x), \Phi_{2}(x)\right)\right) d \partial G\right) \\
& -\mathrm{E}\left(\int_{\gamma}\left(\frac{\partial u_{1}(x)}{\partial V_{A}} \Phi_{1}(x)+\frac{\partial u_{2}(x)}{\partial V_{A}} \Phi_{2}(x)\right) d \gamma\right) \\
& =\mathrm{E}\left(\int_{G}\left(W_{1}(x) \Phi_{1}(x)+W_{2}(x) \Phi_{2}(x)\right) d x\right),
\end{aligned}
$$

$$
F\left(u_{1}(x), \Phi_{1}(x)\right)=H\left(u_{2}(x), \Phi_{2}(x)\right)=0
$$

(In Dirichlet problem $u(x)=0$.) By comparison of two sides, we get the system $(2.1)$, with constraints (2.2). This completes the proof.

Now, we formulate the control problem with adding the control and we determine the cost functional.

\subsection{Formulation of the Optimal Control Problem}

Here, we formulate the problem and establish the necessary and sufficient conditions for the optimal control of distributed type. The space $\left[L^{2}(\Omega, \mathrm{F}, P ; G)\right]^{2}$ being the space of controls. For a control $y=\left(y_{1}, y_{2}\right) \in\left[L^{2}(\Omega, \mathrm{F}, P ; G)\right]^{2}$, the state process of the system $u=\left\{u_{1}(y), u_{2}(y)\right\}$ is given by the solution of the following system:

$$
\left\{\begin{array}{cc}
-\Delta u_{1}(y)+a u_{1}(y)-b u_{2}(y)=W_{1}+y_{1} & \text { in } G_{1} \\
u_{1}(y)=0, u_{2}(y)=0 & \text { on } \partial G \quad(2.7) \\
-\Delta u_{2}(y)+b u_{1}(y)+a u_{2}(y)=W_{2}+y_{2} & \text { in } G_{2},
\end{array}\right.
$$

with conditions (2.2). The observation equation is given by $\chi(y)=\left\{\chi_{1}(y), \chi_{2}(y)\right\} \equiv u(y)$, the cost functional is given by:

$$
C(y)=\mathrm{E}\left(\int_{G}\left(\left(u_{1}(y)-\chi_{1 d}\right)^{2}+\left(u_{2}(y)-\chi_{2 d}\right)^{2}\right) d x\right)+\int_{\Omega}\left(\int_{G}\left(M\left(y_{1}^{2}+y_{2}^{2}\right)\right) d x\right) d p,(2.8)
$$

where $\chi_{d}=\left\{\chi_{1 d}, \chi_{2 d}\right\}$ in $\left[L^{2}(\Omega, \mathrm{F}, P ; G)\right]^{2}$.

Then, the control problem is defined by:

$$
\left\{\begin{array}{cc}
y \in Y_{a d} & \text { suchthat } \\
C(z)=\inf C(y) \quad \forall z \in Y_{a d},
\end{array}\right.
$$

where $Y_{a d}$ is a closed convex subset from $\left[L^{2}(\Omega, \mathrm{F}, P ; G)\right]^{2}$.

Since the cost functional (2.8) can be written as:

$$
\begin{aligned}
& C(y)=\mathrm{E}\left(\int_{G}\left(\left(\left(u_{1}(y)-u_{1}(0)+\left(u_{1}(0)-\chi_{1 d}\right)\right)^{2}\right) d x\right)\right. \\
& +\mathrm{E}\left(\int_{G}\left(\left(\left(u_{2}(u)-u_{2}(0)+\left(u_{2}(0)-\chi_{2 d}\right)\right)^{2}\right) d x\right)\right.
\end{aligned}
$$




$$
+\int_{\Omega}\left(\int_{G} M\left(z_{1}^{2}+z_{2}^{2}\right) d x\right) d p
$$

where

$$
\Pi(y, z)=\mathrm{E}\left(\int_{G}\left(\left(u_{1}(y)-u_{1}(0)\right)^{2}+\left(u_{1}(z)-u_{1}(0)\right)^{2}\right) d x\right)+\int_{\Omega} \int_{G}\left(M\left(z_{1}^{2}+z_{2}^{2}\right)\right) d x d p,(2.9)
$$

$M>0$ is a positive constant, then

$$
L(z)=\mathrm{E}\left(\int_{G}\left(-u_{1}(0)+\chi_{1 d}\right)\left(u_{1}(i)-u_{1}(0)\right)+\left(-u_{2}(0)+\chi_{2 d}\right)\left(u_{2}(i)-u_{2}(0)\right) d x\right),(2.10)
$$

and $\Pi(y, y)$ is a stochatic coercive on $\left[L^{2}(\Omega, \mathrm{F}, P ; G)\right]^{2}$. Since $L(z)$ is continuous on $\left[L^{2}(\Omega, \mathrm{F}, P ; G)\right]^{2}$, then there exists a unique optimal control from the general theory in [8]. Moreover, we have the following theorem which gives the characterization of the optimal control.

Theorem 2.2. If the state $u(y)$ is given by (2.1), (2.2) and if the cost functional is given by (2.8), then there exists a unique optimal control $y=\left(y_{1}, y_{2}\right) \in Y_{a d}$ such that $C(y) \leq C(z) \forall z \in Y_{a d}$; Moreover, it is characterized by:

$$
\left\{\begin{array}{cc}
-\Delta h_{1}(y)+a h_{1}(y)+b h_{2}(y)=u_{1}(y)-\chi_{1 d} & \text { in } G_{1} \\
h_{1}(y)=0, h_{2}(y)=0 & \text { on } \partial G \\
-\Delta h_{2}(y)-b h_{1}(y)+a h_{2}(y)=u_{2}(y)-\chi_{2 d} & \text { in } G_{2},
\end{array}\right.
$$

with constraints

$$
\left\{\begin{array}{cc}
\left\{\frac{\partial h_{1}(y)}{\partial V_{A}^{*}}\right\}^{ \pm}=t\left[h_{1}(y)\right],\left\{\frac{\partial h_{2}(y)}{\partial V_{A}^{*}}\right\}^{ \pm}=t\left[h_{2}(y)\right] & \text { on } \gamma \\
{\left[\frac{\partial h_{1}(y)}{\partial V_{A}^{*}}\right]=0,\left[\frac{\partial h_{2}(y)}{\partial V_{A}^{*}}\right]=0} & \text { on } \gamma \\
{\left[h_{1}(y)\right]=\left[h_{2}(y)\right]=0} & \text { on } \gamma,
\end{array}\right.
$$

where $h(y)=\left\{h_{1}(y), h_{2}(y)\right\}$ is the adjoint state process.

Proof. Since $C(y)$ is differentiable and $Y_{a d}$ is bounded, then the optimal control $z$ is characterized (see e.g [8,9]). Using equations (2.9), (2.10), we get

$$
\Pi(y, z-y) \geq L(z-y),(2.11)
$$

and

$$
\begin{aligned}
& \Pi(y, z-y)-L(z-y) \\
& =\mathrm{E}\left(\int_{G}\left(\left(u_{1}(y)-u_{1}(0)\right)\left(\left(u_{1}(z-y)-u_{1}(0)\right)\right)\right) d x\right) \\
& +\mathrm{E}\left(\int_{G}\left(\left(u_{2}(y)-u_{2}(0)\right)\left(\left(u_{2}(z-y)-u_{2}(0)\right)\right)\right) d x\right) \\
& -\mathrm{E}\left(\int_{G}\left(\left(\left(u_{1}(0)-\chi_{1 d}\right)\left(\left(u_{1}(z-y)-u_{1}(0)\right)\right)\right)\right) d x\right)
\end{aligned}
$$




$$
\begin{aligned}
& \left.+\mathrm{E}\left(\int_{G}\left(\left(\left(u_{2}(0)-\chi_{2 d}\right)\left(u_{2}(z-y)-u_{2}(0)\right)\right)\right)\right) d x\right) \\
& +\int_{\Omega}\left(\int_{G} M y_{1}\left(z_{1}-y_{1}\right)+M y_{2}\left(z_{2}-y_{2}\right) d x\right) d p \\
& \left.=\int_{\Omega}\left(\int_{G} M y_{1}\left(z_{1}-y_{1}\right)\right)+M y_{2}\left(z_{2}-y_{2}\right) d x\right) d p \\
& +\mathrm{E}\left(\int_{G}\left(\left(u_{1}(y)-\chi_{1 d}\right)\left(u_{1}(z)-u_{1}(y)\right) d x\right)\right. \\
& +\mathrm{E}\left(\int_{G}\left(\left(u_{2}(y)-\chi_{2 d}\right)\left(u_{2}(z)-u_{2}(y)\right) d x\right) \geq 0,\right.
\end{aligned}
$$

with $\left(B^{*} h(y), u(y)\right)=(h(y), B u(y))$, and $\mathrm{B}$ is defined by:

$$
B \Phi=B\left\{u_{1}(y), u_{2}(y)\right\}=\left(-\Delta u_{1}(y)+a u_{1}(y)-b u_{2}(y),-\Delta u_{2}(y)+b u_{1}(y)+a u_{2}(y)\right) .
$$

Applying the derivative in the sense of distribution, we get

$$
B^{*} h(y)+M^{T} h(y)=u(y)-\chi_{d},
$$

where $B=-\Delta, M=a_{i j}$ and $j=1,2$. So,

$$
\begin{aligned}
& \Pi(y, z-y)-L(z-y) \\
& =\mathrm{E}\left(\int_{G}\left(\left(a h_{1}-b h_{2}\right)\left(u_{1}(z)-u_{1}(y)\right)+\left(b h_{1}+a h_{2}\right)\left(u_{2}(z)-u_{2}(y)\right)\right) d x\right) \\
& +\int_{\Omega}\left(\int_{G}\left(M y_{1}, z_{1}-y_{1}\right)+\left(M y_{2}, z_{2}-y_{2}\right) d x\right) d p \\
& +\mathrm{E}\left(\int_{G}\left(h_{1}\left(-\Delta u_{1}(z)+\Delta u_{1}(y)\right)+\left(h_{2}\right)\left(-\Delta u_{2}(z)+\Delta u_{2}(y)\right)\right) d x\right) \geq 0
\end{aligned}
$$

Hence, from

$\mathrm{E}\left(\int_{G}\left(\left(h_{1}+M y_{1}\right)\left(z_{1}-y_{1}\right)+\left(h_{2}+M y_{2}\right)\left(z_{2}-y_{2}\right)\right) d x\right) \geq 0$

\section{3 $N \times N$ Stochastic Elliptic Systems}

In this section, we generalize the problem (2.1).

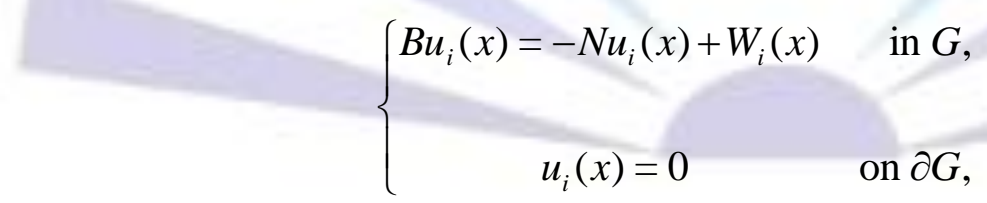

with constraints

$$
\left\{\begin{array}{cc}
{\left[u_{i}(x)\right]=0} & \text { on } \gamma \\
\left\{\frac{\partial u_{i}(x)}{\partial V_{A}}\right\}^{ \pm}=t\left[u_{i}(x)\right] & \text { on } \gamma \\
=0 & \text { on } \gamma, i=1,2, \ldots, n,
\end{array}\right.
$$


where $G$ is an open subset of $\mathrm{R}^{n}$ with smooth boundary, $B$ is an $n \times n$ diagonal matrix of Laplace operators $\left(-\Delta=\sum_{i=1}^{n} \frac{\partial^{2}}{\partial x_{i}^{2}}\right)$, and $N$ is an $n \times n$ matrix with coefficients defined by:

$$
a_{i j}=\left\{\begin{array}{cc}
-1 & i<j, \\
1 & i \geq j .
\end{array}\right.
$$

The model $B$ of the system (2.12) is given by

$$
B u=B\left\{u_{1}, u_{2}, u_{3}, \ldots, u_{n}\right\}=\left\{-\Delta u_{1}+\sum_{j=1}^{n} a_{1 j} u_{j}, \ldots,-\Delta u_{1}+\sum_{j=1}^{n} a_{n j} u_{j}\right\}
$$

On the Sobolev space

$$
\left[H_{0}^{1}(\Omega, \mathrm{F}, P ; G)\right]^{n}=\Pi_{m=1}^{n}\left(H_{0}^{1}(\Omega, \mathrm{F}, P ; G)\right)^{m},
$$

we define the bilinear and linear forms:

$$
\begin{gathered}
b(u, \Phi)=\sum_{i=1}^{n} \mathrm{E}\left(\int_{G}\left(\left(-\Delta u_{i}\right) \Phi_{i} d x+\int_{\gamma}\left(t\left[u_{i}\right]\left[\Phi_{i}\right]\right) d \gamma\right)+\sum_{i, j=1}^{n} \mathrm{E}\left(\int_{G} a_{i j} \Phi_{i} u_{j} d x\right),\right. \\
L(\Phi)=\sum_{i=1}^{n} \mathrm{E}\left(\int_{G} W_{i} \Phi_{i} d x\right),
\end{gathered}
$$

respectively. From (2.3) and (2.14), we have

$$
\begin{aligned}
& b(u, u)=\sum_{i=1}^{n} \mathrm{E}\left(\int_{G}\left|\nabla u_{i}\right|^{2} d x+\int_{\gamma}\left(t\left[u_{i}\right]^{2}\right) d \gamma+\sum_{i=j}^{n} \int_{G}\left|u_{j}\right|^{2} d x\right) \\
& \geq \sum_{i=1}^{n} \mathrm{E}\left(\int_{G}\left|\nabla u_{i}\right|^{2} d x+\sum_{i=j}^{n} \int_{G}\left|u_{j}\right|^{2} d x\right)
\end{aligned}
$$

i.e., $b(u, u)$ is stochastic coercive on $\left[H_{0}^{1}(\Omega, \mathrm{F}, P ; G)\right]^{n}$. Then, by Lax-Milgram lemma, there exists a unique solution $u=\left\{u_{1}, u_{2}, \ldots, u_{n}\right\} \in\left[H_{0}^{1}(\Omega, \mathrm{F}, P ; G)\right]^{n}$ such that $b(u, \Phi)=L(\Phi)$, which proves the existence of the state process for the system (2.10)-(2.12). So, we can formulate the corresponding control problem: The space $\left(L^{2}(\Omega, \mathrm{F}, P ; G)\right)^{n}$ is the space of controls. For a given control $y=\left(y_{i}\right)_{i=1}^{n} \in\left(L^{2}(\Omega, \mathrm{F}, P ; G)\right)^{n}$, the state process $u(y)=\left\{u_{i}(y)\right\}_{i=1}^{n}$ of the system is given by

$$
\left\{\begin{array}{cc}
-\Delta u_{i}(y)=-\sum_{j=1}^{n} a_{i j} u_{i}(y)+W_{i}+y_{i} & \text { in } G_{i} \\
u_{i}(y)=0 & \text { on } \partial G,
\end{array}\right.
$$

where $i=1,2, \ldots, n$, with constraints (2.11). The cost functional is given by:

$$
C(y)=\mathrm{E}\left(\sum_{i=1}^{n} \int_{G}\left(\left(u_{i}(y)-\chi_{i d}\right)^{2} d x\right)+\sum_{i=1}^{n} \int_{\Omega}\left(\int_{G}\left(\left(M\left(y_{i}\right)^{2}\right) d x\right) d p\right.\right.
$$


where

$$
\begin{gathered}
\Pi(y, z)=\mathrm{E}\left(\sum_{i=1}^{n} \int_{G}\left(\left(u_{i}(y)-u_{i}(0)\right)\left(u_{i}(z)-u_{i}(0)\right)\right) d x\right)+\sum_{i=1}^{n} \int_{\Omega}\left(\int_{G}\left(M\left(u_{i}\right)^{2}\right) d x\right) d p, \\
L(z)=\mathrm{E}\left(\sum_{i=1}^{n} \int_{G}\left(\left(-u_{i}(0)+\chi_{i d}\right)\left(u_{i}(z)-u_{i}(0)\right)\right) d x\right),
\end{gathered}
$$

where $\chi_{d}=\left\{\chi_{1 d}, \chi_{2 d}, \ldots, \chi_{n d}\right\}$ in $\left[L^{2}(\Omega, F, P ; G)\right]^{n}$.

In this case, the necessary and sufficient condition for $y$ to be an optimal control is that the following equations and inequalities be satisfied.

$$
\left\{\begin{array}{cc}
B h(y)+N^{T} h(y)=u(y)-\chi_{d} & \text { in } G \\
h_{i}(y)=0 & \text { on } \partial G \\
\left\{\frac{\partial h_{i}(y)}{\partial V_{A}^{*}}\right\}^{ \pm}=t\left[h_{i}(y)\right],\left[\frac{\partial h_{i}(y)}{\partial V_{A}^{*}}\right]=0 & \text { on } \gamma,
\end{array}\right.
$$

where $N^{T}$ is the transpose of $N$ with variational inequality

$$
\sum_{j=1}^{n} \mathrm{E}\left(\int_{G}\left(h_{j}+M y_{j}\right)\left(z_{j}-y_{j}\right) d x\right) \geq 0, \text { where } \frac{\partial h(y)}{\partial V_{A}^{*}}=\sum_{i, k=1}^{n} \cos \left(v, x_{k}\right) \frac{\partial h_{i}(y)}{\partial x_{k}} .
$$

\section{Remark 2.1}

1- If constraints are absent, i.e. when $Y_{a d}=Y$, then $h(z)+M y=0, z_{j} \neq y_{j}$ or $y=-\frac{h(z)}{M}$ the differential problem of finding the vector-function satisfies the the following relations.

For the state process

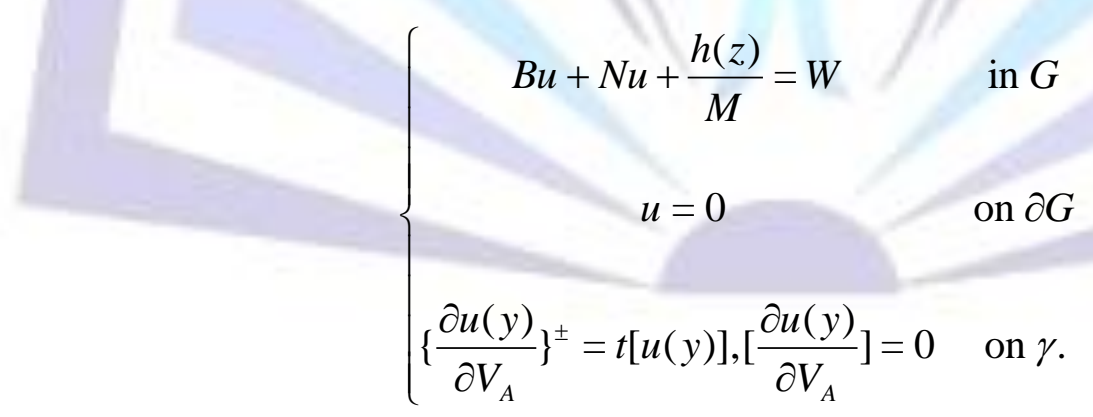

For the adjoint state process 


$$
\left\{\begin{array}{cc}
B h(y)+N^{T} h(y)-u(y)=-\chi_{d} & \text { in } G \\
h(y)=0 & \text { on } \partial G \\
\left\{\frac{\partial h(y)}{\partial V_{A}^{*}}\right\}^{ \pm}=t[h(y)],\left[\frac{\partial h(y)}{\partial V_{A}^{*}}\right]=0 & \text { on } \gamma .
\end{array}\right.
$$

2- We can find the last relations when $N=2$, and get $(2 \times 2)$ stochastic elliptic systems with constraints and distributed Dirichlet conditions.

\section{$32 \times 2$ Neumann Stochastic Elliptic Systems with Constraints}

In this section, we study the optimal control problem for non cooperative $2 \times 2$ stochastic elliptic system with Neumann conditions.

$$
\left\{\begin{array}{cc}
-\Delta u_{1}(x)=W_{1}(x)-a u_{1}(x)+b u_{2}(x) & \text { in } G_{1} \\
\frac{\partial u_{1}(x)}{\partial V_{A}}=g_{1}, \frac{\partial u_{2}(x)}{\partial V_{A}}=g_{2} & \text { on } \partial G \\
\Delta u_{2}(x)=W_{2}(x)-b u_{1}(x)-a u_{2}(x) & \text { in } G_{2},
\end{array}\right.
$$

under constraints

$$
\begin{cases}\left\{\frac{\partial u_{1}(x)}{\partial V_{A}}\right\}^{ \pm}=t\left[u_{1}(x)\right],\left[\frac{\partial u_{1}(x)}{\partial V_{A}}\right]=0 & \text { on } \gamma \\ \left\{\frac{\partial u_{2}(x)}{\partial V_{A}}\right\}^{ \pm}=t\left[u_{2}(x)\right],\left[\frac{\partial u_{2}(x)}{\partial V_{A}}\right]=0 & \text { on } \gamma\end{cases}
$$

where $g \in H^{\frac{1}{2}}(\Omega, \mathrm{F}, P ; G), k=1,2, u=\left\{u_{1}, u_{2}\right\}$.

\subsection{Existence and Uniqueness of Solution}

In this subsection, we study the existence and uniqueness of solutions for $2 \times 2$ non cooperative stochastic systems governed by Neumann problems. Since

$$
\left[H_{0}^{1}(\Omega, \mathrm{F}, P ; G)\right]^{2} \subseteq\left[H^{1}(\Omega, \mathrm{F}, P ; G)\right]^{2}
$$

then

$$
\mathrm{P} u \mathrm{P}_{\left[H_{0}^{1}(\Omega, \mathrm{F}, P ; G)\right]^{2}}^{2} \subseteq \mathrm{P} u \mathrm{P}_{\left[H^{1}(\Omega, \mathrm{F}, P ; G)\right]^{2}}^{2},
$$

which proves the coerciveness of bilinear form $a(u, u)$ on $\left[H^{1}(\Omega, \mathrm{F}, P ; G)\right]^{2}$

$$
b(u, u) \geq c \mathrm{P}_{u} \mathrm{P}_{\left[H^{1}(\Omega, \mathrm{F}, P ; G)\right]^{2}}^{2} \quad \text { (Stochasticcoerciveness) (3.2) }
$$

Theorem

3.1.

Assume that (3.2) holds, and then there exists a unique solution $u=\left\{u_{1}, u_{2}\right\}$ of system (3.1). 
Proof. Since the bilinear form $b(u, \Psi)$ is continuous and stochastic coercive on $\left[H^{1}(\Omega, \mathrm{F}, P ; G)\right]^{2}$, then by Lax Milgram lemma there exist a unique solution of:

$$
b(u, \Psi)=L(\Psi), \forall u \in\left[H^{1}(\Omega, F, P ; G)\right]^{2},
$$

where $L(\Psi)$ is continuous linear form defined on $\left[H^{1}(\Omega, \mathrm{F}, P ; G)\right]^{2}$ by using Green's formula, we obtain (3.1):

$$
L(\Psi)=\mathrm{E}\left(\int_{G}\left(W_{1} \Psi_{1}+W_{2} \Psi_{2}\right) d x+\int_{\partial G}\left(g_{1} \Psi_{1}+g_{2} \Psi_{2}\right) d \partial G\right)
$$

then (3.3) is equivalent to

$$
\begin{aligned}
& b(u, \Psi)=\mathrm{E}\left(\int_{G}\left(\nabla u_{1} \nabla \Psi_{1}+\nabla u_{2} \nabla \Psi_{2}+a u_{1} \Psi_{1}-b u_{2} \Psi_{1}+b u_{1} \Psi_{2}+a u_{2} \Psi_{2}\right) d x\right) \\
& +\mathrm{E}\left(\int_{\gamma}\left(t\left[u_{1}\right]\left[\Psi_{1}\right]+t\left[u_{2}\right]\left[\Psi_{2}\right]\right) d \gamma+\int_{\partial G}\left(\frac{\partial u_{1}(x)}{\partial V_{A}} \Psi_{1}+\frac{\partial u_{2}(x)}{\partial V_{A}} \Psi_{2}\right) d \partial G\right) \\
& =\mathrm{E}\left(\int_{G}\left(W_{1} \Psi_{1}+W_{2} \Psi_{2}\right) d x+\int_{\partial G}\left(g_{1} \Psi_{1}+g_{2} \Psi_{2}\right) d \partial G\right)
\end{aligned}
$$

Hence (3.3) is equivalent to (3.1) and there exists a unique solution of (3.1).

\subsection{Formulation of the Optimal Control Problem with Neumann Conditions}

Here, we formulate the problem and establish necessary and sufficient conditions for the optimal control of distributed type. The space $\left[L^{2}(\Omega, \mathrm{F}, P ; G)\right]^{2}$ is the space of controls. For a control $y \in\left[L^{2}(\Omega, \mathrm{F}, P ; G)\right]^{2}$, the state $u(y)$ of the system is given by the solution of

$$
\left\{\begin{array}{cc}
-\Delta u_{1}(y)=-a u_{1}(y)+b u_{2}(y)+W_{1}(y)+y_{1} & \text { in } G_{1} \\
\frac{\partial u_{1}(y)}{\partial V_{A}}=g_{1}, \frac{\partial u_{2}(y)}{\partial V_{A}}=g_{2} & \text { on } \partial G \\
\left\{\frac{\partial u_{k}(y)}{\partial V_{A}}\right\}^{ \pm}=t\left[u_{k}(y)\right],\left[\frac{\partial u_{k}(y)}{\partial V_{A}}\right]=0, k=1,2 & \text { on } \gamma \\
\Delta u_{2}(y)=-b u_{1}(y)-a u_{2}(y)+W_{2}(y)+y_{2} & \text { in } G_{2} .
\end{array}\right.
$$

The observation is given by $\chi(y)=u(y)$, the cost functional is given again by (3.4). The optimal control is characterize by the following theorem:

Theorem 3.2. Assume that (3.2) holds, if the cost functional is given by (2.7), then there exists an optimal control $y=\left(y_{1}, y_{2}\right) \in\left[L^{2}(\Omega, \mathrm{F}, P ; G)\right]^{2}$. Moreover, it is characterized by the following equations and inequalities: 


$$
\left\{\begin{array}{cc}
-\Delta h_{1}(y)=-a h_{1}(y)-b h_{2}(y)+M \frac{\partial u_{1}(y)}{\partial V_{A}}-\chi_{1 d} & \text { in } G_{1} \\
\frac{\partial h_{1}(y)}{\partial V_{A}^{*}}=0, \frac{\partial h_{2}(y)}{\partial V_{A}^{*}}=0 & \text { on } \partial G \\
\Delta h_{2}(y)=b h_{1}(y)-a h_{2}(y)+M \frac{\partial u_{2}(y)}{\partial V_{A}}-\chi_{1 d} & \text { in } G_{2} .
\end{array}\right.
$$

Under constraints

$$
\left\{\frac{\partial h_{k}(y)}{\partial V_{A}^{*}}\right\}^{ \pm}=t\left[h_{k}(y)\right],\left[\frac{\partial h_{k}(y)}{\partial V_{A}^{*}}\right]=0 \text { on } \gamma, k=1,2 .
$$

Together with (3.4), where $p(u)$ is the adjoint state

$$
\mathrm{E}\left(\int_{G}\left(N y_{1}\left(z_{1}-y_{1}\right)+N y_{2}\left(z_{2}-y_{2}\right)+h_{1}\left(z_{1}-y_{1}\right)+h_{2}\left(z_{2}-y_{2}\right)\right) d x\right) \geq 0
$$

\subsection{Generalization $N \times N$ for Stochastic Neumann Elliptic Systems with Constraints} Here, we generalize the discussion to $N \times N$ stochastic elliptic systems with Neumann condition

$$
\left\{\begin{array}{cc}
A U=-M U+W & \text { in } G \\
\frac{\partial U(x)}{\partial V_{A}}=g & \text { on } \partial G \\
\left\{\frac{\partial U(x)}{\partial V_{A}}\right\}^{ \pm}=t[U(x)],\left[\frac{\partial U(x)}{\partial V_{A}}\right]=0 & \text { on } \gamma
\end{array}\right.
$$

where $G$ is an open subset of $\mathrm{R}^{n}$ with smooth boundary, $A$ is an $N \times N$ diagonal matrix of Laplace operator $\left(-\Delta=\sum_{i=1}^{n} \frac{\partial^{2}}{\partial x_{i}^{2}}\right), M$ is an $N \times N$ matrix with coefficients defined by:

$$
a_{i j}=\left\{\begin{array}{cc}
-1 & i<j, \\
1 & i \geq j .
\end{array}\right.
$$

In this case, the bilinear and linear forms are given by:

$$
\begin{gathered}
b(u, \Psi)=\sum_{i=1}^{n} \mathrm{E}\left(\int_{G}\left(\left(-\Delta u_{i}\right) \Psi_{i} d x+\int_{\gamma}\left(t\left[u_{i}\right]\left[\Psi_{i}\right]\right) d \gamma\right)\right. \\
+\sum_{i, j=1}^{n} \mathrm{E}\left(\int_{G} a_{i j} \Psi_{i} y_{j} d x+\int_{\partial G} \frac{\partial y_{i}(u)}{\partial V_{A}} \Psi_{i} d \partial G\right)
\end{gathered}
$$




$$
L(\Psi)=\sum_{i=1}^{n} \mathrm{E}\left(\int_{G} W_{i} \Psi_{i} d x+\int_{\partial G} g_{i} \Psi_{i} d \partial G\right)
$$

The cost functional is given by:

$$
C(y)=\mathrm{E}\left(\sum_{i=1}^{n} \int_{G}\left(u_{i}(y)-\chi_{i d}\right)^{2} d x\right)+\sum_{i=1}^{n} \int_{\Omega} \int_{G} M\left(y_{i}\right)^{2} d x d p
$$

where $\chi_{d}=\left\{\chi_{1 d}, \chi_{2 d}, \ldots, \chi_{n d}\right\}$ in $\left[L^{2}(\Omega, F, P ; G)\right]^{n}$. In this case the necessary and sufficient condition for $y$ to be an optimal control is that the following equations and inequalities be satisfied.

$$
\left\{\begin{array}{cc}
A h(y)+M^{T} h(y)=M \frac{\partial h(y)}{\partial V_{A}^{*}}-\chi_{d} & \text { in } G \\
\frac{\partial h(u)}{\partial V_{A}^{*}}=0 & \text { on } \partial G \\
\left\{\frac{\partial h(u)}{\partial V_{A}^{*}}\right\}^{ \pm}=t\left[h_{i}(y)\right],\left[\frac{\partial h_{i}(y)}{\partial V_{A}^{*}}\right]=0 & \text { on } \gamma,
\end{array}\right.
$$

where $\frac{\partial h_{i}(y)}{\partial V_{A}^{*}}=\sum_{i, k=1}^{n} \cos \left(v, x_{k}\right) \frac{\partial h_{i}(y)}{\partial x_{k}}, \sum_{j=1}^{n} \mathrm{E}\left(\int_{G}\left(\left(h_{j}+M y_{j}\right)\left(z_{j}-y_{j}\right)\right) d x\right) \geq 0$

Remark 3.1 1 - If constraints are absent, i.e. when $Y_{a d}=Y$, then $h(y)+N y=0$ or $y=-\frac{h(y)}{N}$ the differential problem of finding the vector-function satisfies the following relations:

For the state process

$$
\begin{array}{cc}
A U+M U=W & \text { in } G \\
\frac{\partial U(y)}{\partial V_{A}}+\frac{h(u)}{N}=g & \text { on } \partial G \\
\left\{\frac{\partial U(y)}{\partial V_{A}}\right\}^{ \pm}=t[u(y)],\left[\frac{\partial U(y)}{\partial V_{A}}\right]=0 & \text { on } \gamma
\end{array}
$$

For the adjoint state process

$$
\left\{\begin{array}{cc}
A h(y)+M^{T} h(y)-M \frac{\partial h(y)}{\partial V_{A}^{*}}=-\chi_{d} & \text { in } G \\
\frac{\partial h(y)}{\partial V_{A}^{*}}=0, & \text { on } \partial G \\
\left\{\frac{\partial h(y)}{\partial V_{A}^{*}}\right\}^{ \pm}=t[h(y)],\left[\frac{\partial h_{i}(y)}{\partial V_{A}^{*}}\right]=0 & \text { on } \gamma .
\end{array}\right.
$$


2- We can find the last relations when $N=2$, and get $2 \times 2$ stochastic elliptic systems with constraints and distributed Neumann conditions.

\section{References}

1. A. Al-Hussein, (2009) Backward stochastic partial differential equations driven by infinite dimensional martingales and applications, Stochastics, 81, 6, 601-626.

2. A.Debussche, M.Fuhrman and G.Tessitore, (2007) Optimal control of a stochastic heat equation with boundarynoise and boundary-control. ESAIM Control Optim Calc Var.13, 1, 178-205.

3. B. Øksendal, F. Proske and T. Zhang, (2005) Backward stochastic partial differential equations with jumps and application to optimal control of random jump fields, Stochastics, 77, 5, 381-399.

4. Cla'udio Roberto A'vila da Silva Ju'nior, Milton Kist, and Marcelo Borges dos Santos, (2014) Application of Galerkin Method to Kirchhoff Plates Stochastic Bending Problem ,ISRN Applied Mathematics , 2014.

5. F.Masiero, (2010) A stochastic optimal control problem for the heat equation on the halfline with Dirichlet Boundary-Noise and Boundary-Control. Appl Math Optim.62, 253-294.

6. F.Masiero, (2008) Stochastic optimal control and problems parabolic equations in Banach spaces. SIAM J Control Optim., $47,1,251-300$.

7. J. Ma, J. Yong, (1999) Forward-backward stochastic differential equations and their applications. Lecture Notes in Mathematics, Springer-Verlag, Berlin.

8. Lions, J.L., (1971) Optimal control of a system governed by partial differential equations, Springer -Verlag, New York 170 .

9. Lions, J.L., (1981) Some methods in the mathematical analysis of systems and their control, Sc. Press, Beijing, China.

10. L. Chen and Z. Wu, (2009) Maximum principle for stochastic optimal control problem of forwardbackward system with delay, in Joint 48th IEEE Conference on Decision and Control and 28th Chinese Control Conference, Shanghai, P. R. China .

11. M.Fuhrman, G.Tessitore, (2002) Nonlinear Kolmogorov equations in infinite dimensional spaces: the backward stochastic differential equations approach and applications to optimal control. Ann Probab., 30, 3, 1397-1465.

12. Pontryagin, L.S., et al., (1962) the Mathematical theory of optimal processes, Intersc.

13. P.L.Chow, (1987) Turbulence and Related Problems, in Probabilistic Analysis and Related Topics, 1,edited by A.T.Bharucha-Reid, Academic Press, New York.

14. Sergienko, I.V., Deineka, V.S., (2004) Optimal control of an elliptic system with conjugation conditions and Neumann boundary conditions, Cybernetic and Systems Analysis, 40, 6 .

15. X. Y. Zhou, (1993) On the necessary conditions of optimal controls for stochastic partial differential equations, SIAM J. Control Optim., 31, 6, 1462-1478.

16. [Y. Hu, S. Peng, (1995) Solution of forward-backward stochastic differential equations, Probab. Theory Related Fields, 103, 273-283. 